\title{
NITAZOXANIDE AS A NEW TREATMENT MODALITY ADJUNCTIVE TO NONSURGICAL TREATMENT OF STAGE II PERIODONTITIS: CLINICAL AND BIOCHEMICAL EVALUATION
}

\author{
Maha R. Taalab*, Shaimaa Makled**, Labiba El-Khordagui**, \\ Maha El Tantawi ${ }^{* * *}$ and Sylvia Farid*
}

\begin{abstract}
Objectives: This study evaluated the effect of Nitazoxanide (NTZ) delivered subgingivally as thermosensitive hydrogel on the clinical and biochemical outcomes of scaling and root planing (SRP) compared to SRP alone in patients with stage II periodontitis.

Materials and Methods: A randomized, controlled clinical trial was conducted on forty adult patients equally divided between a control group treated with full-mouth SRP and a test group treated with SRP and sub gingivally-delivered $0.01 \%$ NTZ gel. The gel was administered twice, one week after SRP and a week later. Probing depth (PD), clinical attachment level (CAL), bleeding on probing (BOP), gingival index (GI), and matrix metalloproteinase-8 (MMP-8) levels in gingival crevicular fluid (GCF) were measured at baseline and after 3 and 6 months and compared in addition to the reduction in these values from baseline to 6 months using Mann-Whitney $U$ test.
\end{abstract}

Results: There were significantly greater reductions in the test than the control groups at 6 months regarding PD (mean $=3.1 \mathrm{~mm}$ and $2.3 \mathrm{~mm}, \mathrm{P}=0.001)$, CAL $(2.3 \mathrm{~mm}$ and $1.7 \mathrm{~mm}, \mathrm{P}=0.013)$, percentage of sites with $\mathrm{BOP}($ mean $=1.6 \%$ and $1 \%, \mathrm{P}=0.001)$, $\mathrm{GI}($ mean $=2.3$ and $1.6, \mathrm{P}=0.006$ ), and MMP-8 level (mean= 1.8 and $0.9, \mathrm{P}=0.028)$.

Conclusions: NTZ has potential when combined with SRP as an adjunctive for the treatment of stage II periodontitis, offering added benefit over SRP alone based on clinical and biochemical findings.

Trial registration: The study was retrospectively registered at clinicaltrials.gov, NCT04768530 on $24 / 2 / 2021$.

KEYWORDS: Antimicrobial, Biochemical, Gingival crevicular fluid, Matrix metalloproteinase-8, Periodontitis.

* Department of Oral Medicine, Periodontology, Oral Diagnosis and Oral Radiology, Faculty of Dentistry, Alexandria University, Egypt

** Department of Pharmaceutics, Faculty of Pharmacy, Alexandria University, Egypt

*** Department of Pediatric Dentistry and Dental Public Health, Faculty of Dentistry, Alexandria University, Egypt 


\section{INTRODUCTION}

The oral cavity is a complex ecosystem with multiple distinct niches and microbial habitats. ${ }^{1}$ Changes in microbial flora are associated with oral diseases including periodontitis which is one of the most prevalent oral diseases worldwide affecting a great number of the global population. ${ }^{2}$ Periodontitis is a multifactorial inflammatory condition of the periodontal ligaments with complex interactions between periodontal bacteria,the host inflammatory response, and genetic, environmental, and behavioral risk factors. Periodontitis results in destruction of tooth supporting tissue and eventually, tooth loss. Thus, early effective management of periodontitis is needed. ${ }^{3}$ Tissue destruction is promoted by host immune response involving proinflammatory mediators such as cytokines and matrix metalloproteinases. ${ }^{4}$

Mechanical debridement using SRP is the gold standard treatment for periodontal disease. ${ }^{5}$ Depending on the severity of inflammation, SRP has been combined with systemic antibiotics with variable degrees of success. ${ }^{6}$ This variation is caused by lack of evidence to guide adjunctive systemic antimicrobial therapy including indications, selection of antimicrobial agents or their combinations, proper timing, adequate dosing, and duration of therapy. ${ }^{7}$, ${ }^{8}$ Indiscriminate use of antibiotics led to adverse effects including drug resistance. ${ }^{9}$ Accordingly, there is a need for more pathogen-selective adjunctive therapies that target harmful periodontal anaerobic pathogens.

Recently, research has focused on the topical administration of antimicrobial agents in periodontal pockets as an adjunct to periodontal treatment. Different substances including metronidazole, tetracycline and chlorhexidine have been tested for intrapocket application either alone or in combination with SRP. ${ }^{10}$ Metronidazole is a semiselective antimicrobial that specifically inhibits oral anaerobic bacteria. However, a study reported that $25 \%$ metronidazole gel does not improve probing depth over SRP alone in sites with recurring chronic inflammation. ${ }^{10}$ Metronidazole was also combined with other systemic antibiotics such as amoxicillin. However, several side effects have been reported for this combination. ${ }^{11}$ More recently, the NTZ class of synthetic antibiotics, including the FDA-approved antiparasitic NTZ and its Amixicile analogue, emerged as new antimicrobial agents . NTZ is used for the treatment of parasitic infections such as Cryptosporidium, Trichomonas, Entamoeba, and Giardia. It also shows a distinctive activity against anaerobic bacteria via a unique mechanism of action that defeats mutation-based drug resistance. ${ }^{12,13}$ This mechanism involves the disruption of the enzymatic pyruvate: ferredoxin/ flavodoxin oxidoreductase (PFOR) cycle, which is necessary for energy metabolism in anaerobic bacteria, by targeting the activated thiamine pyrophosphate cofactor rather than the enzyme. ${ }^{14,15}$ Due to the lack of PFOR, killing of commensal or aerotolerant bacteria and, therefore, toxic effects are reduced. ${ }^{15}$ The genome of periodontal pathogenic bacteria includes strict anaerobes containing genes encoding PFOR. Thus, NTZ antimicrobials offer new modalities for controlling periodontitis. In addition, NTZ exhibits anti-inflammatory properties $^{16,17}$ potentially contributing to positive patient outcomes. Although supporting in vitro data are available for the antimicrobial effect of amixicile, ${ }^{13,18}$ the effect of NTZ as a selective antimicrobial agent in treating periodontitis has not been documented to date. Periodontitis is a localized disease, and local drug delivery may enhance the efficacy of adjunctive antimicrobial agents by securing high drug concentration in the periodontal lesion while avoiding systemic side effects. ${ }^{19}$ Using local (intrapocket) sustained-release delivery systems in the form of injectable, thermosensitive hydrogels offers advantage for drug delivery over SRP alone in the management of periodontitis..$^{20,21}$ These formulations exist in a low viscosity sol form at room temperature allowing easy application and fast penetration into periodontal pockets and 
undergo gelation at the higher pocket temperature leading to prolonged site retention, controlled drug delivery, and sustained antimicrobial effect. The efficacy of periodontal therapy is clinically assessed by measuring PD, CAL, BOP, GI, ${ }^{22}$ and biochemical markers including MMPs in the GCF. ${ }^{23}$

The objective of the present study was to assess the clinical and biochemical efficacy of NTZ delivered subgingivally as thermosensitive hydrogel with SRP compared to SRP alone in patients with stage II periodontitis. The null hypothesis was that there would be no difference between NTZ adjunctive to SRP and SRP alone in clinical and biochemical treatment outcomes.

\section{MATERIALS AND METHODS}

\section{Study medication}

The NTZ $0.01 \%$ gel used in the study is a thermosensitive hydrogel consisting of a blend of Poloxamer 407 (P407, average MW 13306 g/mol) and hyaluronic acid (HA, Mw 1000-1500 kD, Xi' an Natural Field Bio-Technique Co., Ltd., China) as gelling agents. ${ }^{24}$ NTZ release from the gel was determined at $37^{\circ} \mathrm{C}$ in phosphate buffer saline $\mathrm{pH}$ $7.4 / 0.01 \%$ Tween 80 as release medium for 7 days at $37^{\circ} \mathrm{C}$.

\section{Study design}

A parallel-groups, randomized, controlled clinical trial was conducted between July 2019 and April 2020 on patients recruited from the Department of Oral Medicine, Periodontology, Diagnosis and Oral Radiology, Faculty of Dentistry, Alexandria University with patients equally allocated to test and control groups. Ethical approval was obtained from the Research Ethics Committee at the Faculty of Dentistry, Alexandria University (IRBNO: 00010556IORG: 0008839). The study purpose and methods were explained to the patients and their informed consent was obtained. The study was conducted in accordance with the Helsinki declaration of 1975, as revised in 2013. The study was registered at clinicaltrials.gov NCT04768530 on 24/2/2021.

\section{Participants}

20- to 40-years-old patients were included if they had stage I/II grade $\mathrm{A} / \mathrm{B}$ periodontitis according to the 2017 World Workshop on the Classification of Periodontal and Peri-Implant Diseases and Conditions. ${ }^{25}$ This diagnosis was confirmed by the presence of $\mathrm{PD} \geq 5 \mathrm{~mm}$ and CAL $3-4 \mathrm{~mm}$, BOP and radiographic horizontal bone loss related to the coronal third of the root (15\%-33\%), with no tooth loss due to periodontitis. Only patients who could maintain an O'Leary plaque index $\leq 10$ participated in the study. ${ }^{26}$ Patients were excluded if they had systemic diseases that may affect the treatment outcomes, if they smoked, were pregnant, or received contraindicated medications, chemotherapy, or radiotherapy in the previous year or if they received any periodontal treatment or antibiotics 6 months before the study. To calculate sample size, we used the estimates of PD reduction reported by Deo et al ${ }^{27}$ in their comparison of the effect of locally applied doxycycline hyclate gel with SRP versus SRP alone. Assuming 5\% alpha error and 80\% study power, the calculated ${ }^{28}$ minimal sample size was 19 per group, increased to 20 to make up for loss to follow up. Thus, the required sample size was 40 patients. Patients were randomly assigned using a computergenerated list of random numbers to one of the two groups in blocks of four and the allocation sequence was handed to an assistant not directly involved with the study. Patients were sequentially numbered in a list and the number of each patient was written on an opaque envelope that included identical pieces of paper that were folded after adding the group to which the patient was assigned. On the day of applying the intervention, the assistant retrieved the envelope carrying the patient number, opened it and the intervention assigned to the patient was provided. Only one indicated site per patient was included, thus not all teeth were treated. If more than one tooth was indicated, one was randomly selected for inclusion then it was randomized to test or control groups. 


\section{INTERVENTION}

Patients were randomized into control group treated with SRP only and test group treated with SRP combined with the subgingivally delivered thermosensitive NTZ hydrogel. Thorough SRP was performed using Woodpecker dental ultrasound scaler handpiece HW-3H and Gracey microcurettes (Hu-Friedy ${ }^{\circledR}$, Chicago, IL, USA) for both groups. The clinical measurements were taken one week after performing SRP. The test sites were dried and isolated with cotton rolls and suction and NTZ hydrogel was applied subgingivally by means of a syringe with a bent, blunt-end needle. (Figure 1). The needle was carefully inserted into the periodontal pocket and approximately $0.5 \mathrm{~mL}$ of the gel was applied gently to fill the pocket. The gel was applied up to the gingival margin and excess gel was removed with a sterile gauze to avoid possible systemic effect by swallowing the gel. No local anesthesia was used. After placement of the gel, patients were instructed to follow a strict oral hygiene protocol and were asked not to chew hard or sticky foods at the gel placement sites. Seven days later, the NTZ hydrogel was applied into the same sites. ${ }^{29}$

\section{Outcome assessment}

The clinical and biochemical treatment outcomes were assessed at baseline, after 3 and 6 months. Clinical assessment included PD in mm, CAL in $\mathrm{mm}, \mathrm{BOP}$ within 15 seconds after probing measured as yes or no at 4 sites per tooth and the percentage of sites with BOP was calculated for each patient, ${ }^{30}$ and GI ranging from score 0 for normal gingiva to score 3 for severe gingivitis. ${ }^{31}$ Periodontal measurements were recorded using William's probe graduated in $1 \mathrm{~mm}$ increment and readings were rounded to the nearest $\mathrm{mm}$. All clinical examinations were performed by a blinded clinician who was unaware of the treatment protocol (S.F). The intra-examiner intra-class correlation coefficient for $\mathrm{PD}=0.89$ and for $\mathrm{CAL}=0.91$. The primary outcome for the study was reduction in $\mathrm{PD}$.
Biochemical assessment included the measurement of MMP-8 levels in the GCF in the deepest pocket depth around the treated area using prefabricated paper points. The paper point was inserted after isolation using cotton rolls and high suction till resistance was felt and was kept for 30 seconds. (Figure2) The samples were diluted in phosphate buffer saline up to $1 \mathrm{~mL}$. After $15 \mathrm{~min}$, the paper points were removed, and $500 \mu \mathrm{L}$ were centrifuged at $400 \mathrm{~g}$ for $4 \mathrm{~min}$, then frozen at $-20^{\circ} \mathrm{C}$. Analysis was done using human MMP-8/Neutrophil collagenase ELISA Kit (BioVision Company., Ltd, Shanghai, China) with sandwich-ELISA technique. The microelisa stripplate in the kit is pre-coated with an antibody specific to MMP-8. Horseradish perosidase (HRP)-conjugated antibody specific for MMP8 was added to each Microelisa stripplate well and was incubated after the samples were added. Free components were washed away. The 3,3',5,5'-Tetramethylbenzidine substrate solution was added to each well. Wells that contained MMP-8 and HRPconjugated MMP-8 antibody appeared blue in color and then turned yellow after the stop solution was added. The optical density, proportional to the concentration of MMP-8, was measured spectrophotometrically at a wavelength of $450 \mathrm{~nm}$. The MMP-8 concentration in the samples was determined using a standard curve. MMP-8 as a biochemical indicator was the secondary outcome of the study.

\section{Statistical analysis}

The Kolmogorov-Smirnov test was used to assess the normality of distribution of the study variables. Reduction was calculated as: (value after 6 months - value at baseline). Mann Whitney U test was used to compare the groups at each follow-up period and the reduction due to $f$ the non-normal distribution of the variables. Friedman test was used to compare differences across time in the same group followed by post hoc Dunn's test for pairwise comparisons. Significance was set at 5\%. Data were analyzed using IBM SPSS software package version 20.0 (Armonk, NY: IBM Corp). 


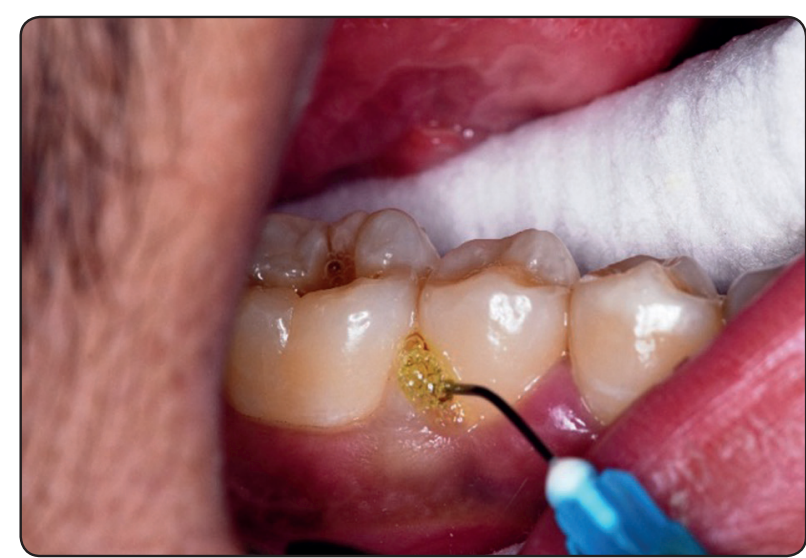

Fig. (1) Subgingival application of the thermosensitive NTZ hydrogel

\section{RESULTS}

\section{Nitazoxanide thermosensitive hydrogel charac- terization}

The in-vitro characterization showed that the NTZ gel (P407 25\% / HA 0.33\%) was a clear lightyellow gel having a $\mathrm{pH}$ 6.7-6.8 and a gelation time $36-38 \mathrm{sec}$. The viscosity of all hydrogels increased over temperatures from $5-37^{\circ} \mathrm{C}$. Free NTZ showed fast dissolution reaching $100 \%$ in $30 \mathrm{~min}$. The gel showed a thermosensitive behavior and sustained the release of NTZ in vitro $(50 \%$ release over 7 days).

\section{Clinical and biochemical parameters}

Out of the 40 patients, 15 (37.5\%) were males and $25(62.5 \%)$ were females and the mean $(\mathrm{SD})$ age in years $=28.9$ (5.6) with no sex or age difference between the two groups $(\mathrm{P}=0.56$ and $0.87)$. The teeth included in the two study groups were premolars and molars: \#4,5,6,7 $(\mathrm{n}=6,8,12$ and 14 respectively, $\mathrm{P}=0.67)$. All participants completed the six-month clinical trial. No adverse reactions were observed in any of the patients from both groups. Patients in the test group reported that the test agent was acceptable to them.

Table 1 shows the change in the clinical and biochemical parameters over time and the difference

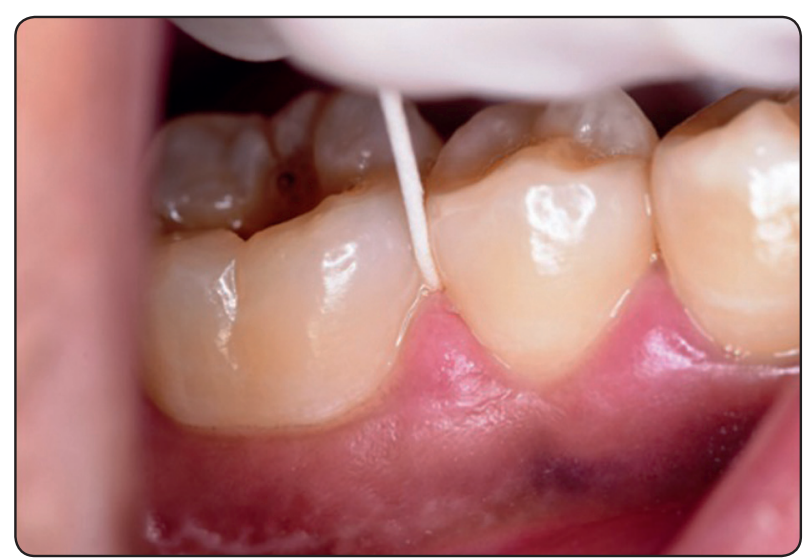

Fig. (2) Gingival crevicular fluid sample collection using a prefabricated paper point

between the two groups at baseline, 3 months and 6 months. At baseline, there were no significant differences between the two groups in $\mathrm{PD}(\mathrm{P}=0.602)$, CAL $(\mathrm{P}=0.429)$, BOP $(\mathrm{P}=1.00)$, GI $(\mathrm{P}=0.799)$ or MMP-8 levels $(\mathrm{P}=0.289)$. PD, CAL, BOP, GI and MMP-8 were significantly reduced over time in both groups $(\mathrm{P}<0.001)$.

For all parameters, there were statistically significant reductions from baseline to 3 and 6 months and from 3 months to 6 months $(\mathrm{P}<0.05)$ except for BOP where the difference between 3 and 6 months in the test group $(P=0.206)$ and the control group $(\mathrm{P}=0.133)$ was not statistically significant.

At all follow-up intervals, there were statistically significant differences between the two groups $(\mathrm{P}<$ $0.05)$ for all parameters except for BOP at 3 months $(\mathrm{P}=0.072)$ and MMP-8 levels at 3 months $(\mathrm{P}=$ 0.925). At 6 months, the test sites had significantly lower parameters than the control sites including $\mathrm{PD}$ (mean $=2.7 \mathrm{~mm}$ and $3.5 \mathrm{~mm}, \mathrm{P}<0.001$ ), CAL (mean $=1.2 \mathrm{~mm}$ and $1.7 \mathrm{~mm}, \mathrm{P}=0.023$ ), BOP (mean $=5 \%$ and $40 \%, \mathrm{P}<0.001$ ), GI (mean $=0.05$ and $0.40, \mathrm{P}<0.001)$ and MMP-8 (mean $=0.5 \mathrm{pg} / \mathrm{ml}$ and $0.9 \mathrm{pg} / \mathrm{ml}, \mathrm{P}=0.033$ ). There were statistically significant differences between the two groups in these reduction from baseline to 6 months in all parameters. 
TABLE (1): Comparison between the two groups in PD, CAL, BOP, GI and MMP-8 levels over the study period

\begin{tabular}{|c|c|c|c|}
\hline & \multicolumn{2}{|c|}{ Mean \pm SD } & \multirow{2}{*}{$\begin{array}{c}\text { P of } \\
\text { MWU } \\
\text { test }\end{array}$} \\
\hline & $\begin{array}{c}\text { Tests } \\
(\mathrm{n}=20)\end{array}$ & $\begin{array}{l}\text { Control } \\
(\mathrm{n}=20)\end{array}$ & \\
\hline \multicolumn{4}{|l|}{ Probing Depth (in mm) } \\
\hline Baseline & $5.7 \pm 0.5$ & $5.8 \pm 0.4$ & 0.602 \\
\hline 3 months & $3.6 \pm 0.9$ & $4.6 \pm 1.2$ & 0.014 \\
\hline 6 months & $2.7 \pm 0.6$ & $3.5 \pm 0.6$ & $<0.001$ \\
\hline P of Friedman test & $<0.001$ & $<0.001$ & \\
\hline Reduction after 6 months & $3.1 \pm 0.6$ & $2.3 \pm 0.5$ & 0.001 \\
\hline \multicolumn{4}{|c|}{ Clinical Attachment Loss (in mm) } \\
\hline Baseline & $3.5 \pm 0.5$ & $3.3 \pm 0.5$ & 0.429 \\
\hline 3 months & $1.6 \pm 0.5$ & $2.1 \pm 0.6$ & 0.046 \\
\hline 6 months & $1.2 \pm 0.5$ & $1.7 \pm 0.5$ & 0.023 \\
\hline $\mathrm{P}$ of Friedman test & $<0.001$ & $<0.001$ & \\
\hline Reduction after 6 months & $2.3 \pm 0.6$ & $1.7 \pm 0.7$ & 0.013 \\
\hline \multicolumn{4}{|l|}{ Bleeding on Probing $\%$} \\
\hline Baseline & $100.00 \pm 0.00$ & $100.00 \pm 0.00$ & 1.00 \\
\hline 3 months & $52.50 \pm 27.98$ & $68.75 \pm 13.75$ & 0.072 \\
\hline 6 months & $5.00 \pm 10.26$ & $40.00 \pm 18.85$ & $<0.001$ \\
\hline $\mathrm{P}$ of Friedman test & $<0.001$ & $<0.001$ & \\
\hline Reduction after 6 months & $1.6 \pm 0.6$ & $1.0 \pm 0.5$ & 0.001 \\
\hline \multicolumn{4}{|l|}{ Gingival Index } \\
\hline Baseline & $2.55 \pm 0.51$ & $2.50 \pm 0.51$ & 0.799 \\
\hline 3 months & $0.58 \pm 0.34$ & $0.86 \pm 0.29$ & 0.011 \\
\hline 6 months & $0.05 \pm 0.10$ & $0.40 \pm 0.19$ & $<0.001$ \\
\hline $\mathrm{P}$ of Friedman test & $<0.001$ & $<0.001$ & \\
\hline Reduction after 6 months & $2.3 \pm 0.7$ & $1.6 \pm 0.7$ & 0.006 \\
\hline \multicolumn{4}{|l|}{ MMP-8 level (pg/ml) } \\
\hline Baseline & $2.2 \pm 1.3$ & $1.81 \pm 1.5$ & 0.289 \\
\hline 3 months & $1.3 \pm 0.8$ & $1.7 \pm 2.1$ & 0.925 \\
\hline 6 months & $0.5 \pm 0.2$ & $0.9 \pm 0.7$ & 0.033 \\
\hline $\mathrm{P}$ of Friedman test & $<0.001$ & $<0.001$ & \\
\hline Reduction after 6 months & $1.8 \pm 1.1$ & $0.9 \pm 0.9$ & 0.028 \\
\hline
\end{tabular}

MWU: Mann Whitney $U$ test

\section{DISCUSSION}

The present study showed lower PD, CAL, BOP, GI and MMP-8 in the NTZ + SRP test group than the SRP alone control group with greater reduction after 6 months from the baseline in the test than the control group in all parameters. The differences were statistically significant in PD, CAL and GI after 3 and 6 months. However, the differences in BOP and MMP-8 GCF levels were statistically significant only after 6 months. Thus, the null hypothesis of the study can be rejected. The present findings provide evidence for the first time about the efficacy of NTZ thermosensitive hydrogel as an adjunctive to SRP to treat stage II periodontitis.

In the present study, NTZ was injectable in sol form at room temperature allowing fast penetration into the deep irregularities of periodontal pockets that cannot be accessed by subgingival instrumentation. Thermosetting of the gel at body temperature formed a drug depot with extended intrapocket delivery which may have facilitated the elimination of periodontal pathogens. ${ }^{32} \mathrm{P} 407$ proved to be a useful gelling agent similar to previous biomedical applications including periodontal delivery systems. ${ }^{32}$ Hydrogels containing P407 increased NTZ solubility due to nanomicelle formation ${ }^{33}$ allowing the preparation of $0.01 \%$ clear NTZ hydrogels. This NTZ concentration is above the reported minimum inhibitory concentration for $90 \%$ inhibition of a wide range of anaerobic and aerobic organisms (1-4 mg/L). ${ }^{32}$ Biocompatible and biodegradable high molecular-weight HA was added to the P407 hydrogels and improved their physicochemical and mechanical properties, reduced their erosion and decreased the cytotoxicity to human gingival fibroblasts and promoted their migration. ${ }^{24,34} \mathrm{HA}$ did not change the gelation properties of NTZ-P407 hydrogels, though it increased the hydrogel viscosity and the sustained release of NTZ with reduction in burst effect as a result of HA-induced packing of the P407 micelles. ${ }^{24}$ Based on the in vitro results obtained in 
the present study, a 25\% P407 / 0.33\% HA gel exhibited adequate sol-gel transition, high storage stability at refrigeration temperature in terms of NTZ content and gel viscosity, good injectability and an acceptable taste. Moreover, the hydrogel in the in vitro part of the study showed $\sim 50 \%$ NTZ release in 7 days, presumably forming an intrapocket drug depot that may be supported by the adherence of the hydrogel to the pocket mucosa. However, in vivo drug release might have occurred at a different rate depending on the intra-pocket hydrodynamic conditions and additional drug release due to gel erosion in periodontal fluids.

Clinical improvement in the control group may be attributed to the removal of biofilm via SRP, with reduction in periodontal bacterial load and healing of soft tissues. ${ }^{35}$ The superiority of clinical outcomes in the test group may be ascribed to the selective bactericidal effect of NTZ against anaerobic bacteria which cause periodontitis ${ }^{14,15}$ and to its anti-inflammatory effect. ${ }^{16,17}$ The improved clinical outcomes of SRP by locally-delivered NTZ is in accordance with previous studies of topical antibiotics used for the treatment of periodontitis. A previous study compared the effect of locallydelivered metronidazole gel adjunctive to SRP and SRP alone and reported a significant difference between SRP alone and SRP plus locally delivered metronidazole gel. ${ }^{36}$ Also, another study reported that subgingivally-delivered nano-doxycycline gel as an adjunct to SRP improved both clinical parameters and inflammatory markers up to three months post treatment. ${ }^{37}$

Despite the importance of clinical parameters, biomarkers provide information on disease status and progression. ${ }^{38}$ MMP- 8 plays an important role in the pathogenesis, diagnosis, and treatment of

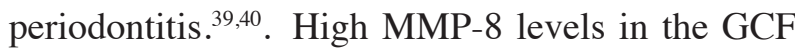
indicate the presence of severe inflammation ${ }^{41}$ and their decrease is associated with effective treatment. ${ }^{42}$ In the current study, patients in both groups showed relatively high GCF MMP-8 levels. ${ }^{43}$ However, the NTZ hydrogel caused significantly greater reduction than the control group in the GCF MMP-8 level after 6 months indicating an anti-inflammatory effect of NTZ not documented to date. NTZ was previously reported to inhibit the production of proinflammatory cytokines such as TNF- $\alpha$, IL-2, IL4 , and IL-10 in peripheral blood by mononuclear cells. ${ }^{17}$ Pro-inflammatory cytokines are responsible for the stimulation of gingival fibroblasts to produce collagenolytic MMPs including MMP$8,{ }^{44}$ and their suppression by NTZ might explain the significant decrease in GCF levels of MMP-8 in the test group. MMP inhibitors whether systemic such as the FDA-approved sub-antimicrobial dose doxycycline or locally-applied like chlorhexidine and proanthocyanidins play an important role as adjuncts in the treatment of periodontitis. ${ }^{45}$ The MMP-8 inhibitory effect of NTZ would add to its selective action against anaerobic bacteria for the treatment of periodontitis.

The clinical and biochemical data obtained in the present study are of practical importance in view of the relatively low NTZ concentration $(0.01 \%)$ and sustained improvement for at least six months. Previous research showed that local antimicrobial adjunctive treatment used higher drug concentrations, e.g. 5-20\% for metronidazole, ${ }^{10,46} 14 \%$ for doxycycline $^{47}$ and $0.5-2.5 \%$ for chlorhexidine. ${ }^{48}$ Moreover, several adjunctive treatments showed plateau level of improvement after three months. ${ }^{45,49}$ The clinical stability observed in the current study may be attributed to site retention of the hydrogel and sustained NTZ delivery. The present study is limited by the length of the follow-up period, the number of patients included and the focus on one type of periodontitis only. Future studies including more participants and longer follow-up during maintenance phase are needed to confirm these findings and give better insight into the role of NTZ in the treatment of different types of periodontitis. 


\section{CONCLUSIONS}

A proof of concept was provided for the clinical benefit of NTZ as a novel adjunctive to SRP in the treatment of stage II periodontitis using a randomized controlled clinical trial on forty patients. A twice application protocol of NTZ formulated as $0.01 \%$ sustained release thermosensitive hydrogel improved the clinical outcomes of SRP over six months due to the selective activity against periodontal anaerobes, MMP-8 inhibitory effect and adequate drug delivery. These findings support the use of NTZ thermosensitive hydrogel as a potential adjunctive to SRP to treat stage II periodontitis.

\section{List of abbreviations}

NTZ: Nitazoxanide

SRT: Scaling and root planing

PD: Probing depth

CAL: Clinical attachment level

GI: Gingival index

BOP: Bleeding on probing

GCF: Gingival crevicular fluid

ELISA: Enzyme-linked immunosorbent assay

MMPs: Matrix metalloproteinases

PFOR: Pyruvate: ferredoxin/ flavodoxin oxidoreductase

HA: Hyaluronic acid

MMP-8: Matrix metalloproteinase-8

HRP: Horseradish peroxide

\section{Declarations}

\section{Ethics approval and consent to participate}

Ethical approval was obtained from the Research Ethics Committee at the Faculty of Dentistry, Alexandria University, Egypt (IRBNO: 00010556IORG:0008839). The objective of the study and the methods used in it were described to the patients and all of them signed an informed consent. The study was performed in accordance with the Helsinki declaration.

\section{Consent for publication}

All patients were informed that this study will be published and signed a consent for publication.

\section{Availability of data and material}

The datasets used and/or analyzed during the current study are available from the corresponding author on reasonable request

\section{Competing interests}

The authors declare that they have no conflict of interest.

\section{Funding}

Not applicable

\section{REFERENCES}

1. Kaan AMM, Kahharova D, Zaura E. Acquisition and establishment of the oral microbiota. Periodontol 2000. 2021 Jun;86(1):123-141. doi: 10.1111/ prd.12366. Epub 2021 Mar 10. PMID: 33690935

2. Bernabe E, Marcenes W, Hernandez CR et al. Global, regional, and national levels and trends in burden of oral conditions from 1990 to 2017: a systematic analysis for the Global Burden of Disease 2017 Study. J Dent Res 2020;99(4):362-373.

3. Ng E, Tay JRH, Balan P, et al. Metagenomic sequencing provides new insights into the subgingival bacteriome and aetiopathology of periodontitis [pubished online ahead of print 6 Jan 2021] J Periodontal Res; doi: 10.1111/jre.12811.

4. Checchi V, Maravic T, Bellini P, et al. The Role of matrix metalloproteinases in periodontal disease. Int $\mathrm{J}$ Environ Res Public Health 2020;17(14):4923.

5. Munasur SL, Turawa EB, Chikte UME, Musekiwa A. Mechanical debridement with antibiotics in the treatment of chronic periodontitis: Effect on systemic biomarkers-A systematic review. Int $\mathrm{J}$ Environ Res Public Health 2020;17(15):5601.

6. Teughels W, Feres M, Oud V, Martin C, Matesanz P, Herrera D. Adjunctive effect of systemic antimicrobials in periodontitis therapy: A systematic review and metaanalysis. J Clin Periodontol 2020;47:257-281 . 
7. Abdallaoui-Maan L, Bouziane A. Effects of timing of adjunctive systemic antibiotics on the clinical outcome of periodontal therapy: A systematic review. J Clin Exp Dent 2020;12:e300-e309.

8. Khattri S, Kumbargere Nagraj S, Arora A, et al. Adjunctive systemic antimicrobials for the non-surgical treatment of periodontitis. Cochrane Database Syst Rev 2020;11:CD012568.

9. Arredondo A, Blanc V, Mor C, Nart J, Leon R. Resistance to beta-lactams and distribution of beta-lactam resistance genes in subgingival microbiota from Spanish patients with periodontitis. Clin Oral Investig 2020;24:4639-4648.

10. Leiknes T, Leknes KN, Böe OE, Skavland RJ, Lie T. Topical use of a metronidazole gel in the treatment of sites with symptoms of recurring chronic inflammation. J Periodontol 2007;78:1538-1544.

11. Kaufmann ME, Lenherr P, Walter C, Wiedemeier DB, Attin T, Schmidlin PR. Systemically administered amoxicillin/ metronidazole versus azithromycin as adjuncts to subgingival instrumentation during non-surgical periodontal therapy. A systematic review. Swiss Dent J 2020;130:572-583.

12. Shakya A, Bhat HR, Ghosh SK. Update on Nitazoxanide: A Multifunctional Chemotherapeutic Agent. Curr Drug Discov Technol 2018;15:201-213.

13. Hutcherson JA, Sinclair KM, Belvin BR, Gui Q, Hoffman PS, Lewis JP. Amixicile, a novel strategy for targeting oral anaerobic pathogens. Sci Rep 2017;7(1):10474.

14. Hoffman PS, Sisson G, Croxen MA, et al. Antiparasitic drug nitazoxanide inhibits the pyruvate oxidoreductases of Helicobacter pylori, selected anaerobic bacteria and parasites, and Campylobacter jejuni. Antimicrob Agents Chemother 2007;51:868-876.

15. Hoffman PS. Antibacterial Discovery: 21st Century Challenges. Antibiotics (Basel) 2020;9:213.

16. Hong SK, Kim HJ, Song CS, Choi IS, Lee JB, Park SY. Nitazoxanide suppresses IL-6 production in LPS-stimulated mouse macrophages and TG-injected mice. Int Immunopharmacol 2012;13:23-27.

17. Rossignol J-F. Nitazoxanide, a new drug candidate for the treatment of Middle East respiratory syndrome coronavirus. J Infect Public Health 2016;9:227-230.

18. Gui Q, Ramsey KW, Hoffman PS, Lewis JP. Amixicile depletes the ex vivo periodontal microbiome of anaerobic bacteria. J Oral Biosci 2020;62:195-204.
19. Abraham A, Raghavan R, Joseph A, Devi MPS, Varghese M, Sreedevi PV. Evaluation of Different local drug delivery systems in the management of chronic periodontitis: A comparative study. J Contemp Dent Pract 2020;21:280-284.

20. Steinberg D, Friedman M. Sustained-release delivery of antimicrobial drugs for the treatment of periodontal diseases: Fantasy or already reality? Periodontol 2000. 2020 Oct;84(1):176-187. doi: 10.1111/prd.12341. PMID: 32844422 .

21. Wang B, Booij-Vrieling HE, Bronkhorst EM, et al. Antimicrobial and anti-inflammatory thermo-reversible hydrogel for periodontal delivery. Acta Biomater 2020;116:259267.

22. Zhang X,HuZ,Zhu X,LiW, Chen J. Treating periodontitis-a systematic review and meta-analysis comparing ultrasonic and manual subgingival scaling at different probing pocket depths. BMC Oral Health 2020;20:176.

23. González-Ramírez J, Serafín-Higuera N, Mancilla MCS, Martínez-Coronilla G, Famanía-Bustamante J, López ALL. Use of biomarkers for the diagnosis of periodontitis. In: Periodontal Disease-Diagnostic and Adjunctive Nonsurgical Considerations: IntechOpen, 2019.

24. Jung Y-s, Park W, Park H, Lee D-K, Na K. Thermo-sensitive injectable hydrogel based on the physical mixing of hyaluronic acid and Pluronic F-127 for sustained NSAID delivery. Carbohydrate Polymers 2017;156:403-408.

25. Caton JG, Armitage G, Berglundh T, et al. A new classification scheme for periodontal and peri-implant diseases and conditions - Introduction and key changes from the 1999 classification. J Periodontol 2018; 89: 1-8.

26. O'Leary, T.J., Drake, R.B. and Naylor, J.E. (1972), The Plaque Control Record. Journal of Periodontology, 43: 3838. https://doi.org/10.1902/jop.1972.43.1.38

27. Deo V, Ansari S, Mandia S, Bhongade M. Therapeutic efficacy of subgingivally delivered doxycycline hyclate as an adjunct to non-surgical treatment of chronic periodontitis. J. Oral Maxillofac. Res. 2011, 2, e3. https://pubmed.ncbi. nlm.nih.gov/24421985/

28. Faul F, Erdfelder E, Buchner A, Lang AG. Statistical power analyses using $\mathrm{G}^{*}$ Power 3.1: tests for correlation and regression analyses. Behav Res Meth 2009;41:1149-1160.

29. Stelzel M, Florès-de-Jacoby L. Topical metronidazole application compared with subgingival scaling. A clinical and microbiological study on recall patients. J Clin Periodontol 1996;23:24-29. 
30. Ainamo J, Bay I. Problems and proposals for recording gingivitis and plaque. Int Dent J 1975;25:229-235.

31. Loe H, Silness J. Periodontal disease in pregnancy. I. prevalence and severity. Acta Odontol Scand 1963;21:533-551.

32. Dubreuil L, Houcke I, Mouton Y, Rossignol JF. In vitro evaluation of activities of nitazoxanide and tizoxanide against anaerobes and aerobic organisms. Antimicrob Agents Chemother 1996;40:2266-2270.

33. Sheshala R, Quah SY, Tan GC, Meka VS, Jnanendrappa N, Sahu PS. Investigation on solution-to-gel characteristic of thermosensitive and mucoadhesive biopolymers for the development of moxifloxacin-loaded sustained release periodontal in situ gels. Drug Deliv Transl Res 2019;9:434-443.

34. Chen M, Li L, Wang Z, Li P, Feng F, Zheng X. High molecular weight hyaluronic acid regulates $\mathrm{P}$. gingivalis-induced inflammation and migration in human gingival fibroblasts via MAPK and NF- $\varkappa \mathrm{B}$ signaling pathway. Arch Oral Biol 2019;98:75-80.

35. Nesbit SP, Gonzalez-Cabezas C, Reside J, et al. Disease control phase of treatment. In: Diagnosis and Treatment Planning in Dentistry: Elsevier, 2017:192-225. e191.

36. Al-Mubarak SA, Karring T, Ho A. Clinical evaluation of subgingival application of metronidazole $25 \%$, and adjunctive therapy. J Int Acad Periodontol 2000;2:64-70.

37. Madi M, Pavlic V, Samy W, Alagl A. The anti-inflammatory effect of locally delivered nano-doxycycline gel in therapy of chronic periodontitis. Acta Odontol Scand 2018;76:71-76.

38. Al-Majid A, Alassiri S, Rathnayake N, Tervahartiala T, Gieselmann D-R, Sorsa T. Matrix metalloproteinase-8 as an inflammatory and prevention biomarker in periodontal and peri-implant diseases. Int J Dent 2018;2018:7891323.

39. Hernandez M, Baeza M, Contreras J, et al. MMP-8, TRAP-5, and OPG levels in GCF diagnostic potential to discriminate between healthy patients' mild and severe periodontitis sites. Biomolecules 2020;10:1500.

40. Sorsa T, Tjäderhane L, Konttinen YT, et al. Matrix metalloproteinases: contribution to pathogenesis, diagnosis and treatment of periodontal inflammation. Ann Med 2006;38:306-321.
41. Mauramo M, Ramseier AM, Mauramo E, et al. Associations of oral fluid MMP-8 with periodontitis in Swiss adult subjects. Oral Dis 2018;24:449-455.

42. Hernández M, Gamonal J, Tervahartiala T, et al. Associations between matrix metalloproteinase- 8 and -14 and myeloperoxidase in gingival crevicular fluid from subjects with progressive chronic periodontitis: a longitudinal study. J Periodontol 2010;81:1644-1652.

43. Aleksandrowicz P, Żelechowska P, Agier J, et al. Evaluation of metalloproteinase-8 levels in crevicular fluid of patients with healthy implants or periodontitis. Mediators Inflamm 2017;2017:4920847.

44. Cox S, Eley B, Kiili M, Asikainen A, Tervahartiala T, Sorsa $T$. Collagen degradation by interleukin- $1 \beta$-stimulated gingival fibroblasts is accompanied by release and activation of multiple matrix metalloproteinases and cysteine proteinases. Oral Dis 2006;12:34-40.

45. Boelen G-J, Boute L, d'Hoop J, EzEldeen M, Lambrichts I, Opdenakker G. Matrix metalloproteinases and inhibitors in dentistry. Clin Oral Investig 2019;23:2823-2835.

46. Nastri L, De Rosa A, De Gregorio V, Grassia V, Donnarumma G. A new controlled-release material containing metronidazole and doxycycline for the treatment of periodontal and peri-implant diseases: Formulation and in vitro testing. Int J Dent 2019;2019.

47. Dannewitz B, Lippert K, Lang NP, Tonetti MS, Eickholz P. Supportive periodontal therapy of furcation sites: non-surgical instrumentation with or without topical doxycycline. J Clin Periodontol 2009;36:514-522.

48. Zhao H, Hu J, Zhao L. Adjunctive subgingival application of Chlorhexidine gel in nonsurgical periodontal treatment for chronic periodontitis: a systematic review and metaanalysis. BMC Oral Health 2020;20:34.

49. Rudhart A, Purucker P, Kage A, Hopfenmüller W, Bernimoulin JP. Local metronidazole application in maintenance patients. Clinical and microbiological evaluation. J Periodontol 1998;69:1148-1154. 